Sylvatic

A form of disease that occurs in wild animals.
* Laboratory of Infectious Diseases, National Institute of Allergy and Infectious Diseases, National Institutes of Health, Bethesda, Maryland 20892, USA ${ }^{\ddagger}$ Center for Immunization Research, Department of International Health, Johns Hopkins Bloomberg School of Public Health, Baltimore, Maryland 21205, USA. Correspondence to S.S.W. e-mail:

swhitehead@niaid.nih.gov doi:10.1038/nrmicro1690

Published online 11 June 2007

\title{
Prospects for a dengue virus vaccine
}

\section{Stephen S. Whitehead*, Joseph E. Blaney*, Anna P. Durbin ${ }^{\ddagger}$ and Brian R. Murphy*}

Abstract | The number of cases of severe dengue disease continues to grow in endemic areas of southeast Asia, Central and South America, and other subtropical regions. Children bear the greatest burden of disease, and the development of an effective vaccine remains a global public health priority. A tetravalent vaccine is urgently needed and must be effective against all four dengue virus serotypes, be cost-effective and provide long-term protection. In this Review we discuss the unique immunological concerns in dengue virus vaccine development and the current prospects for the development of an acceptable vaccine, a goal that is likely to be reached in the near future.

The dengue viruses (DENV) (BOX 1) are members of the Flavivirus genus of the Flaviviridae family, which includes several viruses that pose a threat to public health, including yellow fever virus (YFV), West Nile virus (WNV), Japanese encephalitis virus (JEV) and tick-borne encephalitis virus $(\mathrm{TBEV})^{1}$. Each of the four serotypes of DENV (DENV-1, DENV-2, DENV-3 and DENV-4) is capable of causing the full spectrum of clinical manifestations following DENV infection, ranging from an asymptomatic infection to dengue fever (DF) and the most severe disease, dengue haemorrhagic fever (DHF) and dengue shock syndrome (DSS). DENV causes significantly more human disease than any other arbovirus, as evidenced by the estimated 50-100 million DENV infections and hundreds of thousands of cases of DHF and DSS that occur annually, with children bearing much of the disease burden ${ }^{2,3}$. DHF and DSS remain a leading cause of hospitalization and death of children in at least eight southeast Asian countries ${ }^{2}$.

Prior to the 1970s, only five countries located in southeast Asia had reported $\mathrm{DHF}^{4}$. However, $\mathrm{DHF}$ has now been documented in $>60$ countries, and DENV is endemic in $>100$ countries, including most of southeast Asia, southern and central America, the Caribbean and South Pacific regions $^{2}$ (FIG. 1). DENV is present in Africa, but intense disease outbreaks are rarely reported, which may be partly due to limited surveillance ${ }^{5}$. Co-circulation of each serotype is common within the countries that are most affected by $\mathrm{DENV}^{6}$. This rapid and intense spread of DENV to most of the tropical and subtropical world has lead to its classification as an emerging infectious disease and has resulted in intensified efforts to prevent infection.

DENV is transmitted to humans through the bite of an infected Aedes mosquito ${ }^{7}$. Aedes aegypti, a highly domesticated mosquito, is by far the predominant vector of DENV, but Aedes albopictus can also sustain transmission ${ }^{8}$.
The dramatic increase in the incidence of disease caused by DENV in the Americas over the past three decades is mainly due to the geographical expansion of Ae. aegypti after the decline of vector control efforts ${ }^{9}$. DENV is unusual among the arboviruses in that it does not require an enzootic cycle for maintenance of epidemic transmission in humans (FIG. 2). Rather, the virus is maintained by a human-to-mosquito-to-human cycle of transmission. A sylvatic cycle of transmission does exist in the forests of Asia and western Africa between non-human primates and Aedes mosquitoes, but the contribution of this enzootic cycle to urban epidemic transmission is believed to be minimal ${ }^{7}$.

\section{Clinical disease}

The World Health Organization (WHO) has provided case definitions for DF, DHF and DSS caused by DENV ${ }^{2}$ (BOX 2). Infection by DENV can result in a range of clinical manifestations, from inapparent infection to DF, DHF and DSS ${ }^{2,10,11}$. Although classic DF has been recognized for $>200$ years $^{12}$, DHF and DSS has only recently been recognized as a clinical syndrome, the first reports being made in the $1950 \mathrm{~s}^{13}$. The degree of vascular leak and haemorrhagic manifestations generally differentiate these clinical syndromes. Atypical clinical presentations can include fulminant haemorrhagic disease with organ failure and encephalopathy.

Classic dengue fever. In general, the majority of DENV infections are either asymptomatic or only mildly symptomatic ${ }^{10,14}$, although conditions have occasionally favoured a more symptomatic presentation of the disease in the infected population, as seen in Tahiti in 1971 (REF. 15). Most symptomatic infections present as classic $\mathrm{DF}$, with an incubation period that can range from 3 to 14 days but is generally 4 to 7 days ${ }^{16-20}$. DF presents with 


\begin{abstract}
Box 1 | The dengue virus genome and virus particle
Dengue virus (DENV)

contains a single-stranded, positive-sense RNA genome. The DENV genome

comprises approximately 10,600 nucleotides and is capped by a $5^{\prime}$-type 1 structure $\left(\mathrm{m}^{7} \mathrm{G} 5^{\prime} \mathrm{ppp} 5^{\prime} \mathrm{A}\right)$, but it does not contain a polyadenylated $3^{\prime}$-tail sequence ${ }^{101}$. Both the $5^{\prime}$ - and $3^{\prime}$-untranslated regions (UTRs) have key roles in the regulation of translation and genomic RNA synthesis ${ }^{102}$. Translation of viral RNA yields a single polypeptide that is co-translationally processed by viral and cellular proteases, generating three structural proteins and at least seven non-structural (NS) proteins (see the figure, part a).

The DENV virion is a spherical, enveloped virus

that has a diameter of approximately $50 \mathrm{~nm}^{103}$ (see the figure, part b). The virion contains three structural proteins (capsid (C), membrane (M) and envelope (E)) and the RNA genome. The membrane precursor, prM, is believed to aid in the folding of the E glycoprotein and both are integrated in the lipid bilayer of the virion by two transmembrane regions that surround a nucleocapsid of unknown structure ${ }^{103}$. At a late stage of virion assembly in the trans-Golgi network, prM is cleaved by furin, which results in a rearrangement of the $\mathrm{M}$ and $\mathrm{E}$ proteins on the virion surface yielding mature infectious virions. The surface of the mature DENV virion is smooth with the envelope proteins aligned in pairs parallel to the virion surface ${ }^{104}$. The E glycoprotein mediates cell attachment and fusion and is also the major target of protective antibodies. The E glycoprotein can be divided into three structural or functional domains: the central domain; the dimerization domain which presents a fusion peptide; and the receptor-binding domain. Virions enter cells by receptor-mediated endocytosis, which is followed by fusion of the viral and cellular membranes mediated by the $\mathrm{E}$ protein under acidic conditions within the endosome ${ }^{102}$. Following fusion, the RNA genome dissociates from the viral nucleocapsid and enters the cytoplasm where it functions as mRNA and is translated. Virion assembly occurs in the endoplasmic reticulum and viruses exit the cell through the Golgi network. Part b reproduced with permission from REF. 104 @ Cell Press.
\end{abstract}

a sudden onset of fever accompanied by headache, pain behind the eyes, generalized myalgias and arthralgias, flushing of the face, anorexia, abdominal pain and nausea. It is generally believed that, following inoculation into the skin by a feeding mosquito, the virus replicates in local dendritic cells, with subsequent systemic infection of macrophages and lymphocytes, followed by entry of the virus into the bloodstream. Although DENV varies greatly in its ability to produce viraemia in patients of all ages, high-titre viraemia $\left(\sim 10^{5}-10^{6}\right.$ infectious units per $\mathrm{ml}$ ) occurs during the febrile period, seeding other areas such as the liver ${ }^{21}$. Viraemia following vaccination with live attenuated virus vaccines will need to be much lower than this to be free of disease symptoms. Rash is common in DF, with a reported incidence in some studies of $>80 \%$ and it can be evanescent and polymorphic in its appearance ${ }^{16,17,22}$. The rash can present as macular, maculopapular, morbilliform, scarlatiniform or petechial in character and is most commonly seen on the trunk, the insides of the arms and thighs, and the plantar and palmar surfaces. Intense pruritus and desquamation of the rash can occur. DF is typically milder in younger children compared with older children and adults ${ }^{23-25}$. Leukopenia, thrombocytopenia and elevation in serum transaminases are common laboratory abnormalities reported in symptomatic DENV infection ${ }^{16,26-28}$ and, along with rash, can be seen in some recipients of acceptably attenuated live virus vaccine candidates. A full recovery from DF can be expected, although some dengue infections resulting in atypical severe disease without manifestations of DHF or DSS have been fatal.

DHF and DSS. The more severe forms of DENV infection, DHF and DSS, are characterized by fever, thrombocytopenia, haemorrhagic manifestations and evidence of increased vascular permeability with leakage of intra-

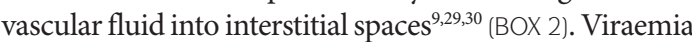
is generally $10-100$-fold greater in DHF and DSS than in $\mathrm{DF}^{31,32}$. DHF/DSS is primarily a disease found in children under the age of 15 in hyper-endemic areas in which all four serotypes of DENV are circulating ${ }^{2,19}$. The haemorrhagic manifestations of DHF include: capillary fragility; petechiae, ecchymoses or purpura; bleeding from the mucosa, gastrointestinal tract or other sites; 


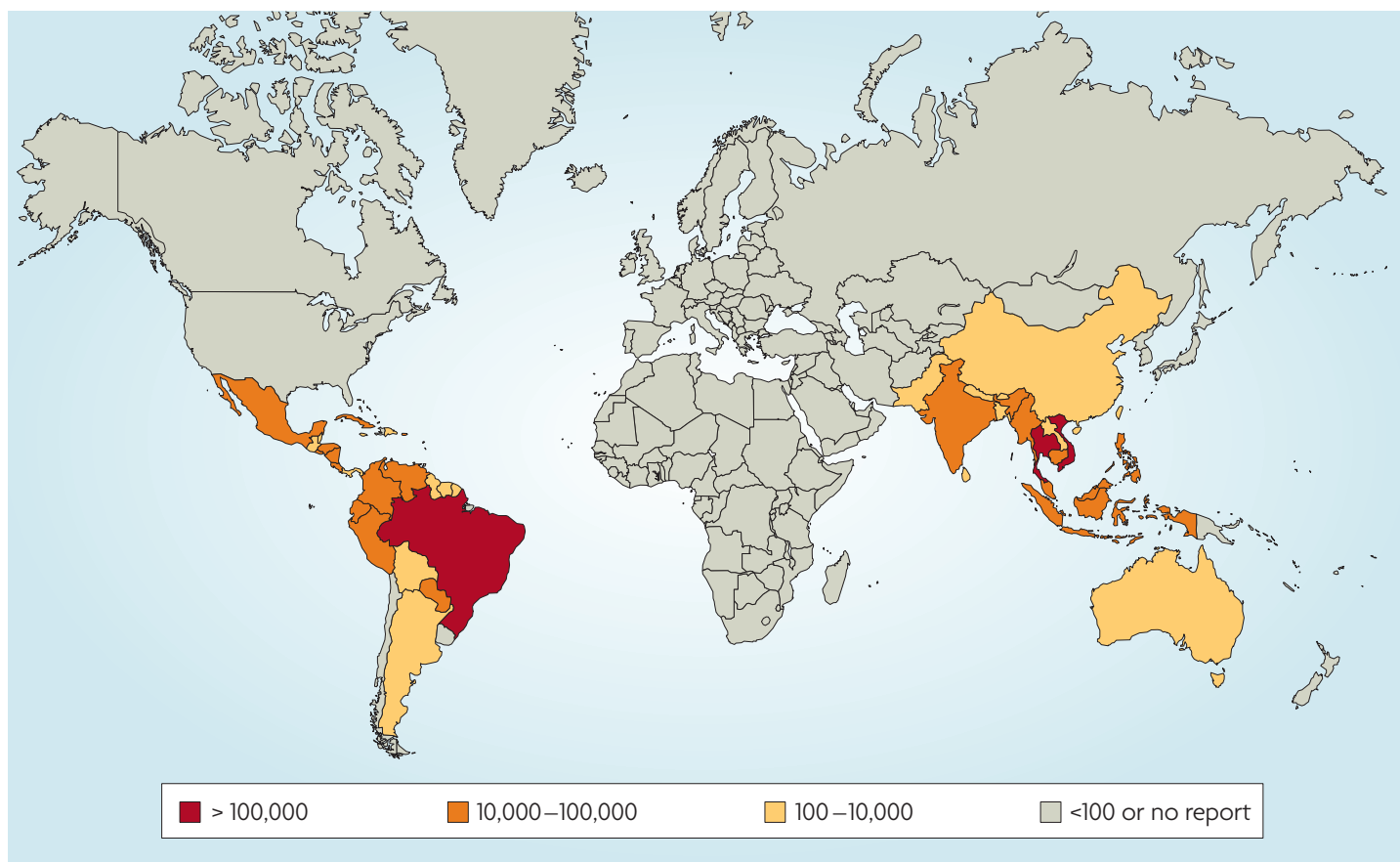

Figure 1 | A decade of dengue disease 1995-2005. Dengue virus (DENV) is endemic in most of the world's tropical and subtropical areas. The number of dengue fever and dengue haemorrhagic fever cases during a single year are shown. A lack of dengue surveillance in Africa during the past decade has made it difficult to assess the level of DENV endemicity in this region. Information taken from WHO DengueNet and PAHO.

Hypovolemia

A decrease in intravascular number.

\section{Ascites}

The accumulation of fluid in the peritoneal cavity.

\section{Councilman bodies}

An eosinophilic globule seen in the liver of individuals with viral haemorrhagic fevers, especially yellow fever. Councilman bodies result from necrosis of a single liver cell.

Adaptive immune response Represented by B and T lymphocytes that express antigen-specific receptors Memory lymphocytes persist, providing immunity against reinfection. and haematemesis or melena. The clinical course of DHF resembles classical DF in its initial presentation of abrupt onset of high fever and other non-specific constitutional signs and symptoms. Around the time of defervescence, the patient's condition suddenly deteriorates, with the advent of haemorrhagic manifestations with or without symptoms of hypovolemia due to plasma leakage. Increased vascular permeability allows the loss of plasma into the interstitial spaces resulting in pleural effusion, a finding that is not always accompanied by haemorrhagic manifestations. Ascites is also common, with a reported incidence of $>50 \%$ in DHF patients examined by ultrasound procedures ${ }^{33,34}$. Hypovolemic shock ensues when sufficient leakage of plasma into the interstitial spaces has occurred. The course of shock is short, but life-threatening, and patients usually succumb or recover within 24 hours. Fulminant liver failure and neurological manifestations have been described in patients with dengue $e^{35-38}$. Hepatomegaly is evident in a high percentage of children with DHF in Thailand, where up to $98 \%$ of children with DHF were reported to have liver enlargement ${ }^{19,30,39}$. Histological examination of the liver has shown marked steatosis, hepatitis with necrosis, and Councilman bodies in some of these cases. The pathogenesis of DHF and DSS is complex and still not completely understood. The observed alterations in coagulation and vascular permeability are believed to arise from a combination of increased virus replication; increased death of cells from infection or cytotoxic immune cells or antibodies; complement activation; and increased release of inflammatory mediators by infected cells or immune cells. The role of the immune response in DHF/DSS is discussed below.

\section{Immunity to DENV infection}

The adaptive immune response to DENV infection contributes to the resolution of infection and has a major role in protection from re-infection. Conversely, it is also believed to have a crucial role in the enhancement of disease severity seen in patients with DHF or DSS. Therefore, immunization against DENV disease must address both the issues of protective immunity and the proposed pathogenic role of antibodies in patients with DHF or DSS (BOX 3 and FIG. 3). It is clear that the presence of neutralizing antibodies directed against the virus envelope (E) protein is the main mediator of protection against DENV infection, and induction of protective levels of neutralizing antibodies is therefore the major goal of immunization. Both live attenuated vaccines and non-living vaccines, such as inactivated virus vaccines, virus-like particles or DNA vaccines, each readily induce both neutralizing antibodies and protective immunity. Barriers to successful immunization, such as the inability to induce long-lasting protective immunity, that exist for hepatitis $\mathrm{C}$ virus and HIV, are not shared with DENV. Robust neutralizing antibody responses develop after DENV infection and are believed to provide lifelong protection against re-infection with the same DENV serotype and shortlived protection of only a few months duration against a heterologous DENV serotype ${ }^{16,40,41}$ (BOX 3). This short period of crossprotection has been associated with the presence of crossreactive neutralizing antibodies that wane rapidly after infection ${ }^{42}$; however, the exact mediator of this protection has not been identified. The role of DENV-specific cellular immunity in protection against 


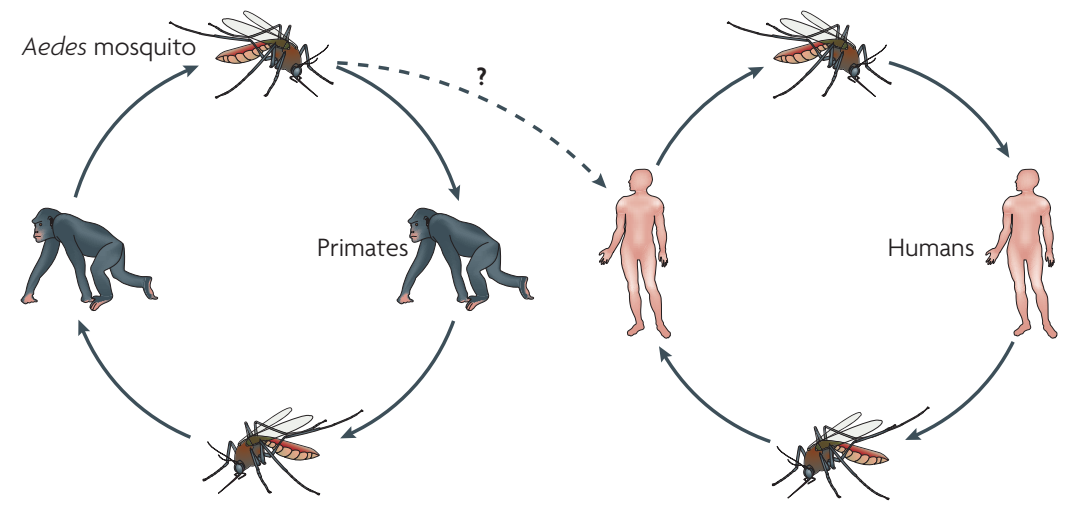

Figure 2 | Transmission of dengue viruses. Because of the high level of viraemia resulting from dengue virus (DENV) infection of humans, the viruses are efficiently transmitted between mosquitoes and humans without the need for an enzootic amplification host. DENV is spread principally by the Aedes aegypti mosquito, which breeds in domestic and peridomestic water containers, increasing the frequency of contact between mosquitoes and humans. In addition, a sylvatic cycle for dengue transmission has been documented in western Africa ${ }^{114}$ and southeast Asia ${ }^{115}$. However, unlike the impact that widespread sylvatic transmission of yellow fever virus has on human disease, the contribution of the observed sylvatic cycle of dengue transmission to human infection is unknown, but appears to be minimal.

re-infection appears to be minor ${ }^{43}$, yet T-cell-mediated immunity to DENV, by analogy to WNV, is likely to contribute significantly to viral clearance ${ }^{44-46}$, and additional research is needed to define the role of T-cell-mediated immunity against DENV.

In endemic areas, illness caused by DENV in infants less than six months of age is unusual and indicates that passively transferred maternal antibodies can protect the infant ${ }^{47-49}$. The titre of neutralizing antibody in mothers' sera was found to correlate with the age of the infant at the time of DENV disease onset, and infants with a neutralizing antibody titre of $>1: 10$ were resistant to DENV disease ${ }^{48}$. Antibody-mediated resistance to DENV infection has also been demonstrated experimentally by passive transfer of monoclonal antibodies in mice ${ }^{50,51}$. Monoclonal antibodies against the virus $\mathrm{E}$ protein were found to be protective and were primarily associated with high neutralizing activity, but protective monoclonal antibodies that were non-neutralizing were also identified. Neutralizing antibodies to domain II and domain III of the E protein (BOX 1) primarily block membrane fusion and virus attachment, respectively ${ }^{1,52-54}$ (BOXES 1,3).

Another feature of immunity to DENV that is of considerable interest to vaccine developers is the ability of a second administration of a live attenuated, tetravalent DENV vaccine to infect monkeys in which neutralizing antibodies have been induced by the first dose of vaccine $e^{55}$. When the second dose of vaccine is given 1 month after the first dose, a boosting effect is not seen. However, a robust boost can be achieved when the second dose is administered after a longer interval of approximately 4 months ${ }^{55-57}$. Presumably, the immunological factors that result in short-term heterotypic immunity are effective at preventing infection with the second dose of vaccine given at 1 month but not at 4 months. The ability to re-infect after 4 months in the presence of neutralizing antibody is surprising. Perhaps the ability of DENV to infect using the IgG Fc $\gamma$ receptor (FcyR) allows it to infect a sufficient number of cells in the presence of neutralizing IgG antibody to induce a vigorous secondary immune response. It is possible that the boost in titre to all four DENV serotypes is the result of a breakthrough infection by a single serotype that induces a heterotypic boost in neutralizing antibody to all four serotypes.

The immune response to DENV not only mediates protection from disease, it also appears to be a major factor in the pathogenesis of DHF and DSS (FIG. 3), although other factors such as the virulence of different virus strains and host genetic factors might have a role ${ }^{58-62}$. The exact immunological mechanisms that mediate enhanced disease remain incompletely defined. However, a strong association of severe disease in humans undergoing a heterotypic secondary infection has been established ${ }^{11,14,31,63,64}$. The enhanced disease severity that is observed after secondary infection by a different DENV serotype is believed to be mediated primarily by pre-existing, non-neutralizing heterotypic antibodies that enhance access of DENV to Fc $\gamma$ R-bearing cells (FIG. 3). Such cells presumably would be inefficiently infected in the absence of antibody. This results in an increase in both the total number of FcyR-bearing cells infected and the total amount of virus produced. This increase in virus replication contributes to the increased titre of virus in the blood of DHF and DSS patients $^{31,32}$ (FIG. 3). This phenomenon is termed antibody-dependent enhancement (ADE) ${ }^{65,66}$. Immune activation and extensive tissue injury caused by augmented virus replication, complement activation and apoptosis presumably mediate the pathological events of DHF and DSS ${ }^{67}$.

A role for ADE in the development of DHF or DSS that is independent of the cellular immune response is suggested by the timing of DHF or DSS in infants, as this generally occurs between the ages of 6 and 12 months in endemic areas ${ }^{47,48,68}$. When the maternal antibody titre to DENV decreases below a protective level (by approximately 6 months of age), infants are actually at an increased risk for the development of DHF and DSS for a short window of time, despite the fact that they have never been infected with a DENV and lack DENV-specific cellular immunity. After the complete degradation of maternal antibodies, infants lose their enhanced susceptibility to DHF and DSS. These observations strongly suggest that pre-existing antibodies in the absence of DENV-specific cellular immunity are sufficient to promote the increased virus replication seen in DHF and DSS, mediated in part by $\mathrm{ADE}^{69-72}$ (FIG. 3).

$\mathrm{ADE}$ and the immunopathogenesis of DHF are features of DENV infection that certainly must be considered in a vaccination programme against DENV. However, existing evidence indicates that a vaccine that induces sustained neutralizing antibody responses against each DENV serotype will be effective at preventing disease and will not contribute to enhanced disease. 
Parenteral route

A route other than the gastrointestinal route, such as intradermal. intravenous, subcutaneous or

\section{The ideal vaccine}

The ideal DENV vaccine would be free from significant reactogenicity, would induce the level of protection afforded by infection with any of the four wild-type viruses, and would provide lifelong protection. Weak immune responses that wane below protective levels over time are not acceptable in the context of the severe consequences seen during secondary DENV infections. As many regions with endemic DENV are in developing countries with limited health resources, the vaccine must be economical with minimal or no repeat immunizations. This should be possible to achieve with existing vaccine candidates and vaccine technologies, and two major aspects of DENV immunobiology

\section{Box 2 The range of dengue disease}

The case definitions of dengue fever (DF), dengue haemorrhagic fever (DHF) and dengue shock syndrome (DSS) are indicated as provided by the $\mathrm{WHO}^{2}$. For a diagnosis of $\mathrm{DHF}$ Grade I, each of the four criteria listed in part $\mathbf{b}$ of the figure must be met. There is a contention from some clinicians that this requirement results in an under-reporting of severe dengue disease as a patient with only two or three severe conditions would be classified as having $\mathrm{DF}^{100}$. A generalized time course of the events associated with DF, DHF and DSS is indicated in part $\mathbf{d}$ of the figure. The incubation period before the development of signs of infection generally ranges from 4 to 7 days. Hypovolemic shock can develop during the late stage of disease and usually lasts 1 to 2 days.

\section{a Dengue fever (DF)}

Febrile illness with 2 or more of the following: headache, retro-orbital pain, myalgia, arthralgia, rash, leukopenia, haemorrhagic manifestations; and virus recovery, serological response or temporal occurrence with other cases b Dengue haemorhagic fever (DHF)

Fever $2-7$ days Petechiae, bruising, or $(+)$ tourniquet test

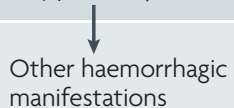

manifestations

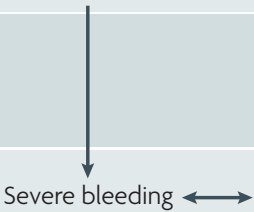

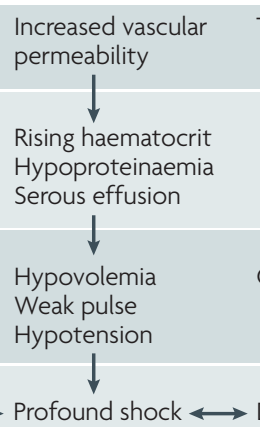

Grade

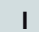

II

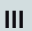

c Dengue shock syndrome (DSS)

Rapid/weak pulse and narrow pulse pressure; or manifestations of hypotension, cold clammy skin and restlessness

d Time course of clinical signs and symptoms

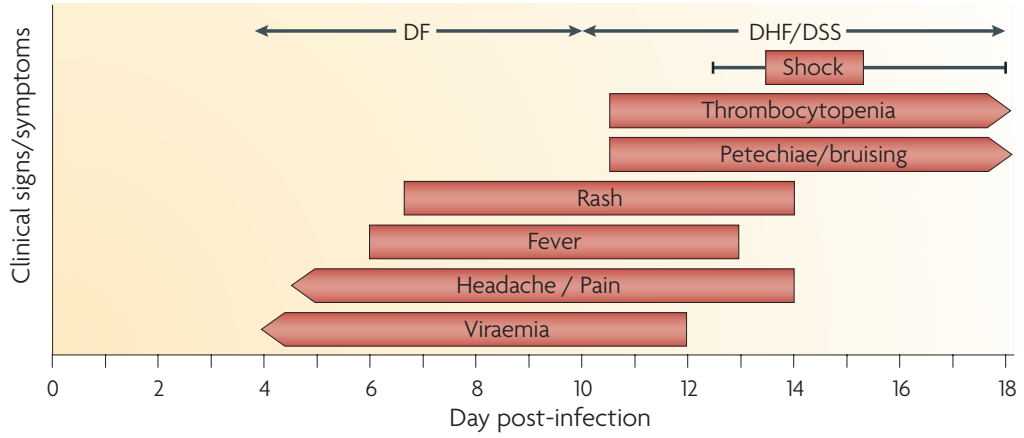

come into play to make this feasible. First, protection is largely mediated by neutralizing antibodies that can be efficiently induced by both live and non-living vaccines. Second, DENV can infect in the presence of preexisting homologous immunity even when the vaccine is administered through a parenteral route, a property that makes it possible to generate secondary antibody responses using a live attenuated virus vaccine. As infection with live attenuated or non-living virus vaccines generally induces less antibody than does infection with wild-type virus, it is highly likely that at least two doses of vaccine will be needed to induce the high levels of antibodies that develop following infection with wild-type DENV. If this goal can be achieved in the short term, then additional booster immunizations years later might not be necessary, as infection with wild-type DENV is thought to provide lifelong protection. However, in non-endemic regions where natural infections are infrequent, consideration should be given to the possible need for additional booster immunizations. Thus, the ideal DENV vaccine could be either a live attenuated tetravalent vaccine given in two doses with a 3-6-month interval between doses, or a non-living virus vaccine, such as inactivated whole virus, subviral particles, a vectored vaccine or a DNA vaccine, given in multiple doses to induce the high levels of long-lived antibodies that develop following wild-type DENV infection.

Live attenuated viruses can be manufactured economically and, as such, are the front-runners in the effort to produce a DENV vaccine. Live attenuated DENV vaccines have been identified that are infectious in humans at doses of $10^{3}$ infectious units ${ }^{73}$. Such vaccines grow to greater than $10^{7}$ infectious units per $\mathrm{ml}$ in Vero cell culture and can induce levels of antibodies following the administration of two doses that are comparable with those achieved following infection with wild-type virus, at least in non-human primates ${ }^{73}$. Hence, a single $100 \mathrm{ml}$ Vero cell culture can yield nearly 1 million doses of vaccine for a single DENV serotype. It remains to be demonstrated that a non-living vaccine can be made that is as immunogenic and as economically feasible as a live attenuated virus. However, if this can be achieved, such a vaccine would be a useful immunogen.

\section{Current vaccine approaches}

Efforts to develop a DENV vaccine have focused mainly on live attenuated virus vaccines, inactivated virus vaccines and subunit virus vaccines. The success of the live attenuated yellow fever (YF) 17D and JEV SA1414-2 vaccines and of the JEV and TBEV inactivated vaccines provides clear guidance for the development of a successful dengue vaccine. Other dengue vaccine approaches, including subunit, vectored and DNA vaccine preparations, are in early development and will only be briefly addressed below. The requirements that four dengue vaccines must be developed and combined as a single vaccine to preclude the development of ADE, DHF and DSS in vaccinees and that the vaccine provides long-term immunity are the major challenges to vaccine development. 


\section{Box 3 | Protective immunity to dengue virus (DENV) infections}

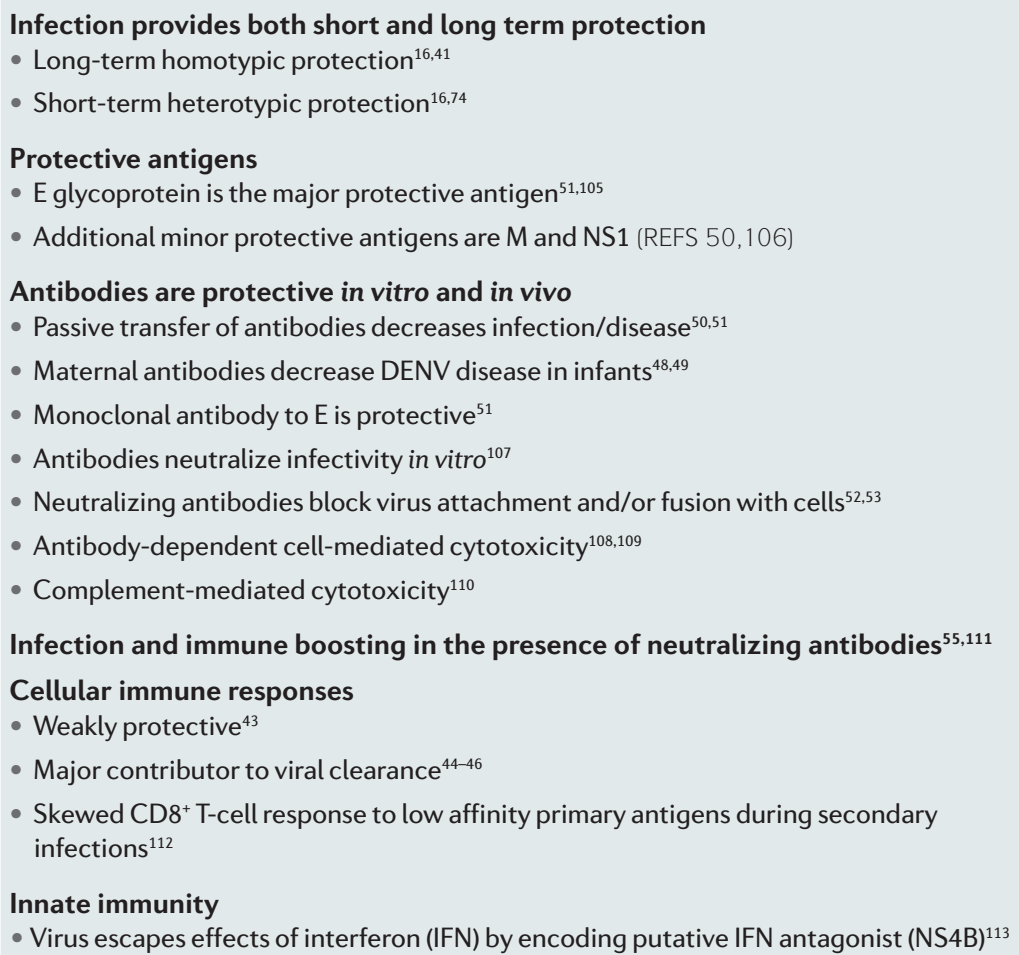

Live attenuated vaccine candidates. Several principles have guided the development of live attenuated vaccine candidates for DENV. First, live virus vaccines can induce durable humoral and cellular immune responses, mimicking natural infection. Live virus vaccines derived from an attenuated DENV should theoretically induce a broad humoral and cellular immune response to both structural and non-structural (NS) proteins, whereas vaccines derived from attenuated antigenic chimeric viruses would induce a more restricted response to the NS proteins, depending on the genetic background (see below). Second, the replication of a live DENV vaccine needs to be sufficiently restricted to preclude the development of significant illness. Symptoms such as fever, headache and arthralgia that are seen in natural dengue disease would generally not be acceptable following vaccination, whereas asymptomatic or sub-clinical signs of infection such as mild rash, mild liver enzyme elevations and transient leukopenia are to be expected as they are part of the normal response to a replicating DENV and would be acceptable. Replication of the vaccine candidate along with its inevitable asymptomatic or subclinical signs of infection is needed to induce a protective immune response, and such is the nature of the delicate balance between vaccine reactogenicity and immunogenicity. A level of viraemia of $10^{1}$ to $10^{2}$ infectious units per $\mathrm{ml}$, as observed following YF vaccination ${ }^{74}$, appears to be a reasonable range for a live DENV vaccine. Third, the virus should have reduced transmissibility by mosquitoes, which can be controlled by a low titred viraemia, such that the 1-2 $\mu \mathrm{l}$ of blood ingested by the mosquito does not contain sufficient virus to initiate an infection or the presence of viral mutations that restrict replication in the mosquito. Fourth, the virus should have high infectivity for humans so that it is infectious at low dose, replicates with reasonable efficiency in tissue culture and can be delivered at low cost. Fifth, each of the four components of the vaccine must replicate sufficiently in humans to induce a balanced neutralizing antibody response to each of the four serotypes. Sixth, it is best if the genetic basis of attenuation for each of the four components is clearly defined so that genetic stability can be monitored during all phases of manufacture and use in humans. The mutations that confer the attenuation phenotype should be genetically and phenotypically stable following replication in humans. With these principles in mind, the live attenuated virus vaccines under development will be discussed.

Mahidol University (Bangkok, Thailand) and the Walter Reed Army Institute of Research (WRAIR) have used conventional methods to develop DENV vaccine candidates by passage in tissue culture cells. As the Mahidol vaccine candidates have not achieved a balanced immune response to each of the four components and systemic symptoms have occurred in recipients of the tetravalent vaccine, this vaccine will not be discussed further ${ }^{75-77}$. The WRAIR candidates were each passaged in primary dog kidney (PDK) cells with terminal passages in fetal rhesus lung cells, and monovalent vaccine candidates have been evaluated in rhesus macaques and in Phase I clinical trials to identify the passage level for each of the PDK-derived vaccine candidates that possess an appropriate balance between attenuation and immunogenicity. In human studies, the DENV-2, DENV-3 and DENV-4 vaccines were only mildly reactogenic, but the DENV-1 candidate retained moderate reactogenicity with $40 \%$ of vaccinees developing fever and generalized rash $^{56}$. Following a single administration of the monovalent candidate vaccines to seronegative adult volunteers, the seroconversion rates ranged from $46-100 \%$ for each DENV serotype, with the highest rate achieved by DENV-1. The under-attenuated nature of the DENV-1 component was also evident in early testing of the tetravalent formulation, which also indicated that the DENV-4 component was slightly over-attenuated $^{56,57}$. To address these issues the dosage levels of each component were adjusted and, eventually, the DENV-1 component was replaced with a further passaged virus (PDK-27 rather than PDK-20) whereas the DENV-4 component was replaced with a lower passaged virus (PDK-6 rather than PDK-20) to improve immunogenicity. Current tetravalent formulations are in Phase II testing in North America and southeast Asia. With the exception of the DENV-2 PDK-53 candidate ${ }^{78}$, the mutations contributing to the attenuation phenotypes of the Mahidol University or WRAIR vaccine candidates have not been identified, partly because the vaccine candidates were never biologically cloned, which has made genetic analysis or re-derivation of the vaccine candidates difficult ${ }^{79}$. 
A separate strategy has been used in the Laboratory of Infectious Diseases at the National Institute of Allergy and Infectious Diseases. Here, reverse genetic techniques have been used to introduce defined attenuating deletion mutations into the $3^{\prime}$-untranslated region (UTR) of DENV-4 and DENV-1 full-length cDNA clones ${ }^{80,81}$. The $3^{\prime} 172-143$ deletion mutation, later referred to as $\Delta 30$, specified a desirable balance between the level of attenuation and immunogenicity for both DENV-1 and DENV-4 in monkeys and humans, but not for DENV-2 and DENV-3 (REFS 82,83). The DENV-1 and DENV-4 vaccine candidates containing the $\Delta 30$ mutation were safe, asymptomatic and immunogenic at $10^{3}$ plaqueforming units (pfu), with a faint rash observed in about half of the volunteers, leukopenia in 7-40\%, and transient increases in alanine aminotransferase (ALT) enzyme levels in a few volunteers, especially at a higher vaccine dose of $10^{5} \mathrm{pfu}$ for DEN $4 \Delta 30$ (REFS 84-86). Vaccine virus was not transmitted from infected vaccinees to mosquitoes probably because the level of viraemia was low $\left(10^{1} \mathrm{pfu}\right.$ per $\left.\mathrm{ml}\right)$ and because the $\Delta 30$ mutation is attenuating for replication in mosquitoes ${ }^{87}$. The $\Delta 30$ mutation remained genetically stable following replication in humans. Because the DEN2 $\Delta 30$ and DEN $3 \Delta 30$ viruses were not suitable vaccine candidates, an alternative chimeric strategy based on the DEN4 $\Delta 30$ vaccine candidate was used to create candidates for DENV-2 and DENV-3. The DEN2/4 330 and DEN3/4 30 vaccine candidates that were generated contained the membrane precursor (prM) and E proteins of DENV-2 or DENV-3 in a DEN4 430 genetic background, and these antigenic chimeric viruses were highly attenuated for monkeys ${ }^{83,88}$. Both the DEN2/4 330 and DEN3/4 30 vaccine candidates have low oral infectivity for Ae. aegypti mosquitoes ${ }^{83,88}$. The attenuation of the DEN2/4 $\Delta 30$ and DEN3/4 $\Delta 30$ viruses was a result of chimerization and the presence of the $\Delta 30$ mutation. The DEN $2 / 4 \Delta 30$ and DEN $3 / 4 \Delta 30$ chimeric vaccine candidates have been combined with DEN $1 \Delta 30$ and DEN $4 \Delta 30$ to create a tetravalent formulation that has been shown to be attenuated (peak titres of $<10^{2}$ pfu per $\mathrm{ml}$ ), broadly immunogenic and protective in rhesus monkeys ${ }^{55}$. Phase I testing of DEN2/4 30 has shown the vaccine candidate to be safe and immunogenic at a dose of $10^{3} \mathrm{pfu}^{89}$, and clinical testing of DEN3/4 330 is currently under way. The suitability of this tetravalent formulation for humans has not yet been studied.

The ChimeriVax platform has been used by Acambis (Cambridge, USA) to create chimeric vaccine candidates by substituting the genes for the prM and E proteins from each of the four DENV serotypes into the live attenuated YF17D vaccine strain. It can be assumed that the attenuation of these vaccine candidates is derived from the mutations present in the YF17D genetic background and chimerization itself, although the contribution of each of these components has not been formally demonstrated ${ }^{90}$. The monovalent vaccine candidates have been shown to have low infection rates for Ae. albopictus, and are essentially non-infectious for Ae. aegypti mosquitoes ${ }^{91}$. In monkeys, administration of a tetravalent formulation of $10^{3}-10^{4.5} \mathrm{pfu}$ of each component induced a high level of neutralizing antibody that was effective against a broad collection of DENV serotypes. However, monkeys experienced a peak viraemia (primarily DENV-4) of $10^{2}-10^{3}$ pfu per ml, which is higher than that observed with other DENV vaccine candidates ${ }^{92}$. The monovalent ChimeriVax-DEN2 vaccine candidate was successfully evaluated in humans and shown to be safe and immunogenic ${ }^{74}$. Early reports of the Phase I testing of the ChimeriVax tetravalent vaccine indicate that it is safe without any serious adverse side effects, but might need to be given at doses greater than $10^{4}$ pfu per ml (sanofi pasteur, unpublished data).

Using the attenuated DENV-2 PDK-53 vaccine strain developed by Mahidol University, researchers at the US CDC have developed a set of chimeric vaccine candidates based on the three attenuating mutations of the DENV-2 PDK-53 strain that exist outside the structural genes. The vaccine candidates have been shown to be immunogenic and protective in mice ${ }^{93}$, and a tetravalent study in monkeys has been recently completed. Preclinical study of the vaccine candidates is ongoing and a Phase I study in humans is anticipated.
Reverse genetic technique A method that allows the production of viruses from cloned CDNA.

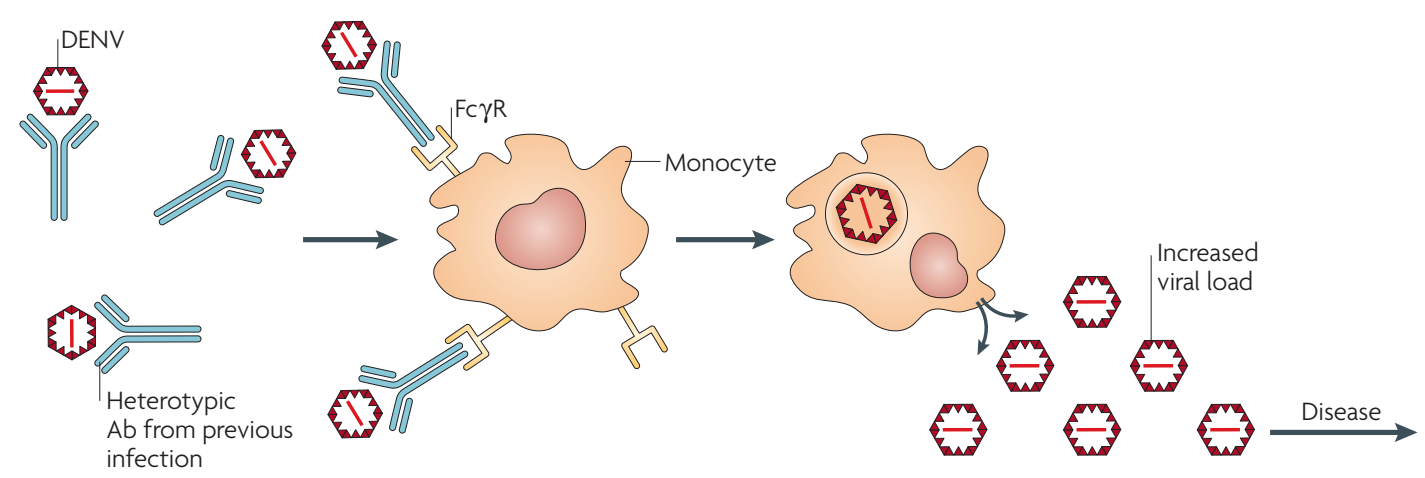

Figure 3 | Model for antibody-dependent enhancement of dengue virus replication. Antibody (Ab)-dependent enhancement of virus replication occurs when heterotypic, non-neutralizing Ab present in the host from a primary dengue virus (DENV) infection binds to an infecting DENV particle during a subsequent heterotypic infection but cannot neutralize the virus. Instead, the Ab-virus complex attaches to the Fc $\gamma$ receptors (Fc $\gamma R$ ) on circulating monocytes, thereby facilitating the infection of $F c \gamma R$ cell types in the body not readily infected in the absence of antibody. The overall outcome is an increase in the overall replication of virus, leading to the potential for more severe disease ${ }^{31}$. 


\begin{tabular}{lllr}
\hline \multicolumn{4}{l}{ Table 1 | A partial list of dengue vaccine candidates that are under development } \\
\hline Vaccine type & Vaccine developer(s) & Clinical testing status & Refs \\
\hline Live attenuated & WRAIR/GSK Biologicals & Tetravalent, Phase II & 57,116 \\
\hline Live attenuated & Mahidol University/sanofi pasteur & No current testing & 77 \\
\hline Live attenuated, chimeric & NIAID, NIH & Monovalent (DENV-1-4), Phase I/II & 73,89 \\
\hline Live attenuated, chimeric & Acambis/sanofi pasteur & Tetravalent, Phase I & 74,92 \\
\hline Live attenuated, chimeric & CDC/InViragen & Preclinical & 93 \\
\hline Inactivated virus & WRAIR & Preclinical & 97 \\
\hline Subunit & Hawaii Biotech & Begins 2007 & 97 \\
DNA & Navy Medical Research Center & Monovalent (DENV-1), Phase I & 99
\end{tabular}

GSK, GlaxoSmithKline; NIAID, National Institute of Allergy and Infectious Diseases; NIH, National Institutes of Health; WRAIR, Walter Reed Army Institute of Research.

Whole virus inactivated vaccines. Inactivated vaccines have two major advantages over live attenuated virus vaccines: safety, as it is not possible for inactivated vaccines to revert to a more pathogenic phenotype; and induction of a balanced antibody response, as each of the four serotypes in a multivalent inactivated virus vaccine should be equally immunogenic. Nonetheless, the use of inactivated whole virus vaccines presents its own challenges: the vaccine only contains the DENV structural proteins and thus fails to induce any immunity to the non-structural proteins; adjuvants are required for optimal immunogenicity in seronegative subjects and these can add expense and reactogenicity to the vaccine; multiple booster doses are required to provide long-term immunity; and they can be expensive to manufacture as DENV does not grow to high titres in tissue culture cells. These challenges make an inactivated DENV vaccine a less attractive vaccine candidate for use in DENVendemic areas, but they might be useful as a military or traveller's vaccine, or as a part of a prime-boost strategy with live or replicating vaccines ${ }^{94}$.

A purified, inactivated DENV-2 vaccine has been manufactured by WRAIR and a DENV-1 equivalent will soon enter clinical trials ${ }^{95,96}$. For preparation of the inactivated vaccine candidates, the viruses were propagated in certified Vero cells, concentrated by ultrafiltration and purified on sucrose gradients. The high-titre purified virus (approximately $10^{9} \mathrm{pfu}$ per $\mathrm{ml}$ ) was then inactivated with formalin. The DENV-2 vaccine with alum and other adjuvants induced high neutralizing antibody levels and protection against viraemia in a primate model $^{97}$.

Recombinant subunit vaccines. Dengue antigens, primarily E proteins, have been produced in several expression systems to generate subunit vaccine candidates. Not surprisingly, most of the subunit preparations elicited moderate to high levels of antibody following immunization of mice. Although such vaccines are anticipated to achieve a high level of safety, it is likely that they will share some or all of the difficulties outlined above for inactivated DENV vaccines. So far, a DENV subunit vaccine has not been tested in humans. However, two studies in rhesus monkeys have recently been completed using monovalent DENV-2 or
DENV-4 truncated E proteins. Guzman et al. immunized monkeys with four doses of $100 \mu \mathrm{g}$ of E protein (DENV-4), using alum as an adjuvant, and achieved only partial protection against wild-type DENV-2 challenge ${ }^{98}$. In collaboration with Hawaii Biotech, Putnak et al. immunized monkeys with two doses of DENV-2 E protein produced in Drosophila cells and formulated with each of five different adjuvant combinations ${ }^{97}$. Although the DENV neutralizing antibody titres before challenge varied widely, one group of monkeys receiving the highest dose of antigen together with two adjuvants was completely protected. Hawaii Biotech is currently manufacturing affinity-purified E protein for each of the four DENV serotypes and will soon initiate a Phase I clinical trial.

Live vectored and nucleic acid vaccines. Although numerous recombinant vector systems have been used to express DENV antigens, the resulting vaccine candidates have achieved limited success using modified vaccinia or adenovirus vectors and additional development of suitable vector platforms will be necessary. Rather than using live vectors to express DENV antigens, such antigens can be expressed from DNA constructs that are introduced into cells and subsequently translated into dengue antigens or subviral particles. In recent experiments using genes encoding prM and E proteins from DENV-1, it was shown that three doses of vaccine protected Aotus monkeys following challenge with wild-type virus ${ }^{99}$. A clinical trial with an improved DENV-1 DNA construct delivered in three doses is currently under way. DNA vaccines afford advantages over conventional vaccines, including ease of production, stability and transport at room temperature, decreased likelihood of replication interference and the possibility to vaccinate against multiple pathogens in a single vaccination. Nonetheless, recent experience with DNA vaccines has highlighted the necessity for multiple doses, experimental adjuvants and immunostimulatory motifs, and specialized injection equipment. Prime-boost strategies using a combination of live vectors or DNA vectors expressing the E protein have been investigated, but such strategies for tetravalent DENV development seem too complicated at present for economical delivery and use in endemic areas. 


\section{Future prospects for vaccines}

A list of vaccine candidates close to, or currently in, clinical evaluation is presented in TABLE 1. Although the pace of vaccine development has recently increased, the challenges facing the development and delivery of an acceptable vaccine to endemic areas are still significant. Little is known about the potential for replication interference among the components of a live attenuated tetravalent vaccine, especially in the context of vaccinees with variable levels of pre-existing immunity. In addition, precise correlates of protection have not been identified, although it is generally assumed that neutralizing antibody has a significant role in protection. Before large-scale vaccine trials are undertaken in endemic regions, the infrastructure and methods must be in place to determine not only the efficacy of the vaccine, but also the safety and immunogenicity of vaccination of both dengue-exposed and dengue-naive individuals, including adults and children. These types of trials will probably also necessitate a re-evaluation of the WHO classification system for dengue disease, which is viewed by many as too rigorous in its assignment of case definitions and uses definitions that are often difficult to fulfill in first-level referral clinics ${ }^{100}$. Guidance documents for the evaluation of a dengue vaccine have been distributed by the WHO, and additional guidelines are being prepared. The capacity for inexpensive vaccine manufacture and distribution is continually changing, especially as the number of local, rather than global, companies step forward to manufacture vaccine in their own countries. Although this type of local manufacture could help to provide vaccine to those who need it most, it is dependent on innovative financing schemes in countries with primarily emerging economies. Although long overdue, the development of a safe and effective dengue vaccine is moving forward at an unprecedented rate, mainly owing to the use of reverse genetics and the availability of Vero cells for manufacture, with a high likelihood that the challenges of vaccine development and implementation can be overcome.
Burke, D. S. \& Monath, T. P. in Fields Virology (eds Knipe, D. M. \& Mowley, P. M.) 1043-1125 (Lippincott, Williams \& Wilkins, Philadelphia, 2001)

2. WHO. Dengue haemorrhagic fever: diagnosis, treatment prevention and control (WHO, Geneva, 1997).

3. Gubler, D. J. \& Meltzer, M. Impact of dengue/dengue hemorrhagic fever on the developing world. Adv. Virus Res. 53, 35-70 (1999).

4. Gubler, D. J. in Dengue and Dengue Hemorrhagic Fever (eds Gubler, D. J. \& Kuno, G.) 1-22 (CAB International, New York, 1997)

5. Thomas, S. J., Strickman, D. \& Vaughn, D. W Dengue epidemiology: virus epidemiology, ecology, and emergence. Adv. Virus Res. 61, 235-289 (2003).

6. Mackenzie, J. S., Gubler, D. J. \& Petersen, L. R. Emerging flaviviruses: the spread and resurgence of Japanese encephalitis, West Nile and dengue viruses. Nature Med. 10, S98-S109 (2004).

A review that highlights the increasing burden of disease associated with DENV and other flaviviruses.

7. Rodhain, F. \& Rosen, L. in Dengue and Dengue Hemorrhagic Fever (eds Gubler, D. J. \& Kuno, G. 45-60 (CAB International, New York, 1997).

8. Effler, P. V. et al. Dengue fever, Hawaii, 2001-2002. Emerg. Infect. Dis. 11, 742-749 (2005).

9. Gubler, D. J. Dengue and dengue hemorrhagic feve Clin. Microbiol. Rev. 11, 480-496 (1998)

10. Endy, T. P. et al. Epidemiology of inapparent and symptomatic acute dengue virus infection: a prospective study of primary school children in Kamphaeng Phet, Thailand. Am. J. Epidemiol. 156 40-51 (2002)

A prospective study describing the range of disease caused by DENV infection.

11. Guzman, M. G. et al. Epidemiologic studies on dengue in Santiago de Cuba, 1997. Am. J. Epidemiol. 152 793-799 (2000)

12. Rush, B. in Medical Inquiries and Observations 89-100 (Prichard and Hall, Philadelphia, 1789).

13. Halstead, S. B., Yamarat, C. \& Scanlon, J. E. The Thai hemorrhagic fever epidemic of 1962: a preliminary report. J. Med. Assoc. Thailand 46, 449-462 (1963).

14. Burke, D. S., Nisalak, A., Johnson, D. E. \& Scott, R. M. A prospective study of dengue infections in Bangkok. Am. J. Trop. Med. Hyg. 38, 172-180 (1988).

15. Moreau, J. P., Rosen, L., Saugrain, J. \& Lagraulet, J. An epidemic of dengue on Tahiti associated with hemorrhagic manifestations. Am. J. Trop. Med. Hyg. 22, 237-241 (1973)
16. Sabin, A. B. Research on dengue during World War II. Am. J. Trop. Med. Hyg 1, 30-50 (1952). Review of studies including virus isolation, experimental infection of humans and analysis of homotypic and heterotypic immunity.

17. Siler, J. F., Hall, M. W. \& Hitchens, A. P. Dengue: its history, epidemiology, mechanism of transmission, etiology, clinical manifestations, immunity, and prevention. Philippine J. Sci. 29, 1-304 (1926).

18. Simmons, J. S., St John, J. H. \& Reynolds, F. H. K Experimental studies of dengue. Philippine J. Sci. 44 1-252 (1931).

19. Eram, S. et al. Epidemic dengue hemorrhagic fever in rural Indonesia: II. clinical studies. Am. J. Trop. Med. Hyg. 28, 711-716 (1979).

20. Halstead, S. B., Nimmannitya, S. \& Margiotta, M. R. Dengue and chikungunya virus infection in man in Thailand, 1962-1964: Il. Observations on disease in outpatients. Am. J. Trop. Med. Hyg. 18, 972-983 (1969).

21. Jessie, K., Fong, M. Y., Devi, S., Lam, S. K. \& Wong, $K$. T. Localization of dengue virus in naturally infected human tissues, by immunohistochemistry and in situ hybridization. J. Infect. Dis. 189 1411-1418 (2004)

22. Lai, P. C. et al. Characteristics of a dengue hemorrhagic fever outbreak in 2001 in Kaohsiung. J. Microbiol. Immunol. Infect. 37, 266-270 (2004)

23. Halstead, S. B. et al. Observations related to pathogenesis of dengue hemorrhagic fever. I. Experience with classification of dengue viruses. Yale Jiol. Med. 42, 261-275 (1970)

24. Halstead, S. B., Scanlon, J., Umpaivit, P. \& Udomsakdi, S. Dengue and chikungunya virus infection in man in Thailand, 1962-1964: IV. Epidemiologic studies in the Bangkok metropolitan area. Am. J. Trop. Med. Hyg. 18, 997-1021 (1969).

25. Innis, B. L. in Kass Handbook of Infectious Diseases: Exotic Virus Infections (ed. Porterfield, J. S.) 103-146 (Chapman \& Hall Medical, London, 1995)

26. Souza, L. J. et al. Aminotransferase changes and acute hepatitis in patients with dengue fever: analysis of 1,585 cases. Braz. J. Infect. Dis. 8, 156-163 (2004).

27. Kalayanarooj, S. et al. Early clinical and laboratory indicators of acute dengue illness. J. Infect. Dis. 176 313-321 (1997)

28. Kuo, C. H. et al. Liver biochemical tests and dengue fever. Am. J. Trop. Med. Hyg. 47, 265-270 (1992).

29. Carlos, C. C. et al. Comparison of clinical features and hematologic abnormalities between dengue fever and dengue hemorrhagic fever among children in the Philippines. Am. J. Trop. Med. Hyg. 73, 435-440 (2005).

30. Nimmannitya, S. Clinical spectrum and management of dengue haemorrhagic fever. Southeast Asian Trop. Med. Public Health 18, 392-397 (1987).
31. Vaughn, D. W. et al. Dengue viremia titer antibody response pattern, and virus serotype correlate with disease severity. J. Infect. Dis. 181 2-9 (2000)

This study indicates that increased disease severity was associated with higher viraemia secondary infection and DENV-2 infection.

32. Murgue, B., Roche, C., Chungue, E. \& Deparis, X. Prospective study of the duration and magnitude of viraemia in children hospitalised during the 19961997 dengue-2 outbreak in French Polynesia. J. Med. Virol. 60, 432-438 (2000).

33 Pramulijo, H. S. \& Harun, S. R. Ultrasound findings in dengue haemorrhagic fever. Pediatr. Radiol. 21, 100-102 (1991)

34. Venkata Sai, P. M., Dev, B. \& Krishnan, R. Role of ultrasound in dengue fever. Br. J. Radiol. 78 , 416-418 (2005).

35. Subramanian, V., Shenoy, S. \& Joseph, A. J. Dengue hemorrhagic fever and fulminant hepatic failure. Dig. Dis. Sci. 50, 1146-1147 (2005).

36. Patey, O., Ollivaud, L., Breuil, J. \& Lafaix, C. Unusual neurologic manifestations occurring during dengue fever infection. Am. J. Trop. Med. Hyg. 48, 793-802 (1993)

37. Nimmannitya, S., Thisyakorn, U. \& Hemsrichart, V. Dengue haemorrhagic fever with unusual manifestations. Southeast Asian J. Trop. Med. Public Health 18, 398-406 (1987)

38. Kho, L. K., Sumarmo, Wulur, H., Jahja, E. \& Gubler, D. J. Dengue hemorrhagic fever accompanied by encephalopathy in Jakarta. Southeast Asian J. Trop. Med. Public Health 12, 83-86 (1981)

39. Wichmann, O. et al. Risk factors and clinical features associated with severe dengue infection in adults and children during the 2001 epidemic in Chonburi, Thailand. Trop. Med. Int. Health 9, 1022-1029 (2004).

40. Guzman, M. G. et al. Dengue hemorrhagic fever in Cuba, 1981: a retrospective seroepidemiologic study. Am. J. Trop. Med. Hyg. 42, 179-184 (1990).

41. Halstead, S. B. Etiologies of the experimental dengues of Siler and Simmons. Am. J. Trop. Med. Hyg. 23, 974-982 (1974)

42. Innis, B. L. in Dengue and Dengue Hemorrhagic Fever (eds Gubler, D. J. \& Kuno, G.) 221-243 (CAB International, Cambridge, 1997).

43. Calvert, A. E., Huang, C. Y., Kinney, R. M. \& Roehrig, J. T. Non-structural proteins of dengue 2 virus offer limited protection to interferon-deficient mice after dengue 2 virus challenge. J. Gen. Virol. 87, 339-346 (2006).

44. Samuel, M. A. \& Diamond, M. S. Pathogenesis of West Nile Virus infection: a balance between virulence, innate and adaptive immunity, and viral evasion. J. Virol. 80, 9349-9360 (2006). 
45. Bukowski, J. F. et al. Dengue virus-specific crossreactive $\mathrm{CD}^{+}{ }^{+}$human cytotoxic $\mathrm{T}$ lymphocytes. J. Virol. 63, 5086-5091 (1989)

46. Kurane, I., Meager, A. \& Ennis, F. A. Dengue virusspecific human T cell clones. Serotype crossreactive proliferation, interferon $\gamma$ production, and cytotoxic activity. J. Exp. Med. 170, 763-775 (1989).

47. Halstead, S. B. et al. Dengue hemorrhagic fever in infants: research opportunities ignored. Emerg. Infect. Dis. 8, 1474-1479 (2002).

48. Kliks, S. C., Nimmannitya, S., Nisalak, A. \& Burke, D. S. Evidence that maternal dengue antibodies are important in the development of dengue hemorrhagic fever in infants. Am. J. Trop. Med. Hyg. 38, 411-419 (1988).

49. Pengsaa, K. et al. Dengue virus infections in the first 2 years of life and the kinetics of transplacentally transferred dengue neutralizing antibodies in thai children. J. Infect. Dis. 194 , 1570-1576 (2006).

\section{References 48 and 49 demonstrate the} presence of DENV-specific maternal antibodies in infants and suggest that they are protective until their levels decrease below a protective threshold.

50. Kaufman, B. M. et al. Monoclonal antibodies for dengue virus prM glycoprotein protect mice against lethal dengue infection. Am. J. Trop. Med. Hyg. 41 576-580 (1989)

51. Kaufman, B. M., Summers, P. L., Dubois, D. R. \& Eckels, K. H. Monoclonal antibodies against dengue 2 virus E-glycoprotein protect mice against lethal dengue infection. Am. J. Trop. Med. Hyg. 36 427-434 (1987)

52. Crill, W. D. \& Roehrig, J. T. Monoclonal antibodies that bind to domain III of dengue virus E glycoprotein are the most efficient blockers of virus adsorption to Vero cells. J. Virol. 75, 7769-7773 (2001)

53. Roehrig, J. T., Bolin, R. A. \& Kelly, R. G. Monoclonal antibody mapping of the envelope glycoprotein of the dengue 2 virus, Jamaica. Virology 246, 317-328 (1998).

54. Roehrig, J. T. in Dengue and Dengue Hemorrhagic Fever (eds Gubler, D. J. \& Kuno, G.) 199-220 (CAB International, New York, 1997)

55. Blaney, J. E., Jr, Matro, J. M., Murphy, B. R. \& Whitehead, S. S. Recombinant, live-attenuated tetravalent dengue virus vaccine formulations induce balanced, broad, and protective neutralizing antibody response against each of the four serotypes in rhesus monkeys. J. Virol. 79, 5516-5528 (2005).

56 . Sun, W. et al. Vaccination of human volunteers with monovalent and tetravalent live-attenuated dengue vaccine candidates. Am. J. Trop. Med. Hyg. 69, 24-31 (2003).

57. Edelman, R. et al. Phase I trial of 16 formulations of a tetravalent live-attenuated dengue vaccine. $\mathrm{Am}$. J. Trop. Med. Hyg. 69, 48-60 (2003)

References $\mathbf{5 6}$ and $\mathbf{5 7}$ describe clinical studies of the empirically derived live attenuated DENV vaccines characterized by the United States Army.

58. Stephens, H. A. et al. HLA-A and-B allele associations with secondary dengue virus infections correlate with disease severity and the infecting viral serotype in ethnic Thais. Tissue Antigens 60, 309-318 (2002).

59. Sakuntabhai, A. et al. A variant in the CD209 promoter is associated with severity of dengue disease. Nature Genet. 37, 507-513 (2005).

60. Fernandez-Mestre, M. T., Gendzekhadze, K. RivasVetencourt, P. \& Layrisse, Z. TNF- $\alpha-308$ A allele, a possible severity risk factor of hemorrhagic manifestation in dengue fever patients. Tissue Antigens 64, 469-472 (2004).

61. Gubler, D. J., Suharyono, W., Lubis, I., Eram, S. \& Gunarso, S. Epidemic dengue 3 in central Java, associated with low viremia in man. Am. J. Trop. Med. Hyg. 30, 1094-1099 (1981).

62. Gubler, D. J., Reed, D., Rosen, L. \& Hitchcock, J. C. Jr. Epidemiological, clinical, and virologic observations on dengue in The Kingdom of Tonga. Am. J. Trop. Med. Hyg. 27, 581-589 (1978)

63. Guzman, M. G. et al. Clinical and serologic study of Cuban children with dengue hemorrhagic fever/dengue shock syndrome (DHF/DSS). Bull. Pan Am. Health Organ. 21, 270-279 (1987)

64. Halstead, S. B., Nimmannitya, S. \& Cohen, S. N. Observations related to pathogenesis of dengue hemorrhagic fever. IV. Relation of disease severity to antibody response and virus recovered. Yale J. Biol. Med. 42, 311-328 (1970).
65. Halstead, S. B. Neutralization and antibodydependent enhancement of dengue viruses. Adv. Virus Res. 60, 421-467 (2003)

A review that discusses the evidence supporting the role of antibody-dependent enhancement in the enhanced severity of DENV infection associated with secondary infection.

66. Halstead, S. B. Pathogenesis of dengue: challenges to molecular biology. Science 239, 476-481 (1988).

67. Kurane, I. \& Ennis, F. A in Dengue and Dengue Hemorrhagic Fever (eds Gubler, D. J. \& Kuno, G.) 273-290 (CAB International, New York, 1997).

68. Nguyen, T. H. et al. Dengue hemorrhagic fever in infants: a study of clinical and cytokine profiles. J. Infect. Dis. 189, 221-232 (2004).

69. Green, S. \& Rothman, A. Immunopathological mechanisms in dengue and dengue hemorrhagic fever. Curr. Opin. Infect. Dis. 19, 429-436 (2006) A broad review of the immunopathological mechanisms that are associated with DENV infection and how they can result in the symptoms of severe disease.

70. Kliks, S. C., Nisalak, A., Brandt, W. E., Wahl, L. \& Burke, D. S. Antibody-dependent enhancement of dengue virus growth in human monocytes as a risk factor for dengue hemorrhagic fever. Am. J. Trop. Med. Hyg. 40, 444-451 (1989).

71. Stephenson, J. R. Understanding dengue pathogenesis: implications for vaccine design. Bull. World Health Organ. 83, 308-314 (2005).

72. Avirutnan, P. et al. Vascular leakage in severe dengue virus infections: a potential role for the nonstructural viral protein NS1 and complement. J. Infect. Dis. 193, 1078-1088 (2006)

73. Blaney, J. E. Jr, Durbin, A. P., Murphy, B. R. \& Whitehead, S. S. Development of a live attenuated dengue virus vaccine using reverse genetics. Viral Immunol. 19, 10-32 (2006)

A summary of the preclinical and clinical progress towards developing a recombinant, tetravalent DENV vaccine at NIAID.

74. Guirakhoo, F. et al. Live attenuated chimeric yellow fever dengue type 2 (ChimeriVax-DEN2) vaccine: Phase I clinical trial for safety and immunogenicity: effect of yellow fever pre-immunity in induction of cross neutralizing antibody responses to all 4 dengue serotypes. Hum. Vaccin. 2, 60-67 (2006). The first reported clinical trial of a monovalent ChimeriVax DENV vaccine candidate.

75. Kanesa-thasan, N. et al. Safety and immunogenicity of attenuated dengue virus vaccines (Aventis Pasteur) in human volunteers. Vaccine 19, 3179-3188 (2001).

76. Sabchareon, A. et al. Safety and immunogenicity of a three dose regimen of two tetravalent liveattenuated dengue vaccines in five- to twelve-year-old Thai children. Pediatr. Infect. Dis. J. 23, 99-109 (2004)

77. Kitchener, S. et al. Immunogenicity and safety of two live-attenuated tetravalent dengue vaccine formulations in healthy Australian adults. Vaccine 24, 1238-1241 (2006).

78. Butrapet, S. et al. Attenuation markers of a candidate dengue type 2 vaccine virus, strain 16681 (PDK-53), are defined by mutations in the $5^{\prime}$ noncoding region and nonstructural proteins 1 and 3. J. Virol. 74, 3011-3019 (2000)

79. Sanchez, V. et al. Innate and adaptive cellular immunity in flavivirus-naive human recipients of a live-attenuated dengue serotype 3 vaccine produced in Vero cells (VDV3). Vaccine 24, 4914-4926 (2006)

80. Men, R., Bray, M., Clark, D., Chanock, R. M. \& Lai, C. J. Dengue type 4 virus mutants containing deletions in the 3' noncoding region of the RNA genome: analysis of growth restriction in cell culture and altered viremia pattern and immunogenicity in rhesus monkeys. J. Virol. 70, 3930-3937 (1996)

81. Whitehead, S. S. et al. A live, attenuated dengue virus type 1 vaccine candidate with a 30-nucleotide deletion in the $3^{\prime}$ untranslated region is highly attenuated and immunogenic in monkeys. J. Virol. 77 1653-1657 (2003)

82. Blaney, J. E. Jr, Hanson, C. T., Hanley, K. A., Murphy, B. R. \& Whitehead, S. S. Vaccine candidates derived from a novel infectious cDNA clone of an American genotype dengue virus type 2. BMC Infect. Dis. 4, 39 (2004)

83. Blaney, J. E. Jr et al. Genetically modified, live attenuated dengue virus type 3 vaccine candidates. Am. J. Trop. Med. Hyg. 71, 811-821 (2004).
84. Durbin, A. P. et al. The live attenuated dengue serotype 1 vaccine rDEN1Delta30 is safe and highly immunogenic in healthy adult volunteers. Hum. Vaccin. 2, 167-173 (2006).

85. Durbin, A. P. et al. rDEN4 $\Delta 30$, a live attenuated dengue virus type 4 vaccine candidate, is safe, immunogenic, and highly infectious in healthy adult volunteers. J. Infect. Dis. 191, 710-718 (2005).

86. Durbin, A. P. et al. Attenuation and immunogenicity in humans of a live dengue virus type- 4 vaccine candidate with a 30 nucleotide deletion in its 3 '-untranslated region. Am. J. Trop. Med. Hyg. 65, 405-413 (2001).

87. Troyer, J. M. et al. A live attenuated recombinant dengue- 4 virus vaccine candidate with restricted capacity for dissemination in mosquitoes and lack of transmission from vaccinees to mosquitoes. Am. J. Trop. Med. Hyg. 65, 414-419 (2001).

88. Whitehead, S. S. et al. Substitution of the structural genes of dengue virus type 4 with those of type 2 results in chimeric vaccine candidates which are attenuated for mosquitoes, mice, and rhesus monkeys. Vaccine 21, 4307-4316 (2003).

89. Durbin, A. P. et al. rDEN2/4 330 (ME), a live attenuated chimeric dengue serotype 2 vaccine is safe and highly immunogenic in healthy dengue-naive adults. Hum. Vaccin. 2, 255-260 (2006).

90. Guirakhoo, F. et al. Construction, safety, and immunogenicity in nonhuman primates of a chimeric yellow fever-dengue virus tetravalent vaccine. J. Virol. 75, 7290-7304. (2001).

91. Higgs, S. et al. Growth characteristics of ChimeriVaxDen vaccine viruses in Aedes aegypti and Aedes albopictus from Thailand. Am. J. Trop. Med. Hyg. 75 986-993 (2006).

92. Guirakhoo, F. et al. Viremia and immunogenicity in nonhuman primates of a tetravalent yellow feverdengue chimeric vaccine: genetic reconstructions, dose adjustment, and antibody responses against wild-type dengue virus isolates. Virology 298 , 146-159 (2002)

93. Huang, C. Y. et al. Dengue 2 PDK-53 virus as a chimeric carrier for tetravalent dengue vaccine development. J. Virol. 77, 11436-11447 (2003) This study describes using recombinant technology to produce antigenic chimeric vaccine candidates in the DENV-2 PDK-53 vaccine genetic background.

94. Simmons, M., Porter, K. R., Hayes, C. G., Vaughn, D. W. $\&$ Putnak, R. Characterization of antibody responses to combinations of a dengue virus type 2 DNA vaccine and two dengue virus type 2 protein vaccines in rhesus macaques. J. Virol. 80 , 9577-9585 (2006)

95. Putnak, R. et al. Development of a purified, inactivated, dengue-2 virus vaccine prototype in Vero cells: immunogenicity and protection in mice and rhesus monkeys. J. Infect. Dis. 174, 1176-1184 (1996).

96. Putnak, R. et al. Immunogenic and protective response in mice immunized with a purified, inactivated, Dengue- 2 virus vaccine prototype made in fetal rhesus lung cells. Am. J. Trop. Med. Hyg. 55, 504-510 (1996)

97. Putnak, R. et al. An evaluation of dengue type-2 inactivated, recombinant subunit, and live-attenuated vaccine candidates in the rhesus macaque model. Vaccine 23, 4442-4452 (2005)

98. Guzman, M. G. et al. Induction of neutralizing antibodies and partial protection from viral challenge in Macaca fascicularis immunized with recombinant dengue 4 virus envelope glycoprotein expressed in Pichia pastoris. Am. J. Trop. Med. Hyg. 69, 129-134 (2003).

99. Raviprakash, K. et al. Needle-free Biojector injection of a dengue virus type 1 DNA vaccine with human immunostimulatory sequences and the GM-CSF gene increases immunogenicity and protection from virus challenge in Aotus monkeys. Virology 315, 345-352 (2003).

100. Deen, J. L. et al. The WHO dengue classification and case definitions: time for a reassessment. Lancet 368 170-173 (2006)

A discussion of the issues surrounding the WHO case definition of DHF and its possible role in under-reporting of severe disease. 
101. Lindenbach, B. D \& Rice, C. M. in Fields Virology (eds Knipe, D. M. \& Howley, P. M.) 1043-1125 (Lippincott Williams and Wilkins, Philadelphia, 2001)

102. Clyde, K. \& Harris, E. RNA secondary structure in the coding region of dengue virus type 2 directs translation start codon selection and is required for viral replication. J. Virol. 80, 2170-2182 (2006).

103. Mukhopadhyay, S., Kuhn, R. J. \& Rossmann, M. G. A structural perspective of the flavivirus life cycle. Nature Rev. Microbiol. 3, 13-22 (2005). A clear review of the flavivirus virion structure and the dynamics of virion formation.

104. Kuhn, R. J. et al. Structure of dengue virus. Implications for flavivirus organization, maturation, and fusion. Cell 108, 717-725 (2002).

105. Bray, M. et al. Mice immunized with recombinant vaccinia virus expressing dengue 4 virus structural proteins with or without nonstructural protein NS1 are protected against fatal dengue virus encephalitis. J. Virol. 63, 2853-2856 (1989).

106. Schlesinger, J. J., Brandriss, M. W., Cropp, C. B. \& Monath, T. P. Protection against yellow fever in monkeys by immunization with yellow fever virus nonstructural protein NS1. J. Virol. 60, 1153-1155 (1986).

107. Russell, P. K., Nisalak, A., Sukhavachana, P. \& Vivona, S. A plaque reduction test for dengue virus neutralization antibodies. J. Immunol. 99, 285-290 (1967).

108. Laoprasopwattana, K et al. Dengue virus (DV) enhancing antibody activity in preillness plasma does not predict subsequent disease severity or viremia in secondary DV infection. J. Infect. Dis. 192, 510-519 (2005).
109. Garcia, G. et al. Antibodies from patients with dengue viral infection mediate cellular cytotoxicity. J. Clin. Virol. 37, 53-57 (2006).

110. Falgout, B., Bray, M., Schlesinger, J. J. \& Lai, C. J. Immunization of mice with recombinant vaccinia virus expressing authentic dengue virus nonstructural protein NS1 protects against lethal dengue virus encephalitis. J. Virol. 64, 4356-4363 (1990).

111. Sabchareon, A. et al. Safety and immunogenicity of tetravalent live-attenuated dengue vaccines in Thai adult volunteers: role of serotype concentration, ratio, and multiple doses. Am. J. Trop. Med. Hyg 66, 264-272 (2002)

112. Mongkolsapaya, J. et al. Original antigenic sin and apoptosis in the pathogenesis of dengue hemorrhagic fever. Nature Med. 9, 921-927 (2003)

113. Munoz-Jordan, J. L. et al. Inhibition of $\alpha / \beta$ interferon signaling by the NS4B protein of flaviviruses. J. Virol. 79, 8004-8013 (2005)

114. Diallo, M. et al. Amplification of the sylvatic cycle of dengue virus type 2, Senegal, 1999-2000: entomologic findings and epidemiologic considerations. Emerg. Infect. Dis. 9, 362-367 (2003).

115. Wolfe, N. D. et al. Sylvatic transmission of arboviruses among Bornean orangutans. Am. J. Trop. Med. Hyg. 64, 310-316 (2001).

116. Sun, W. et al. Protection of Rhesus monkeys against dengue virus challenge after tetravalent live attenuated dengue virus vaccination. J. Infect. Dis. $193,1658-1665$ (2006)

\section{Acknowledgements}

Support for S.S.W., J.E.B., A.P.D. (in part) and B.R.M. is provided by the Intramural Research Program of the National Institute of Allergy and Infectious Diseases, National Institutes of Health, USA.

\section{Competing interests statement}

The authors declare competing financial interests: see web version for details.

\section{DATABASES}

The following terms in this article are linked online to:

Entrez Genome: http://www.ncbi.nlm.nih.gov/entrez/query. fcgi?db=genome

DENV |JEV |TBEV |WNV |YFV

Entrez Genome Project: http://www.ncbi.nlm.nih.gov/ entrez/query.fcgi?db=genomeprj Aedes aegypti|Aedes albopictus

\section{FURTHER INFORMATION}

Laboratory of Infectious Diseases:

http://www3.niaid.nih.gov/labs/aboutlabs/lid Mahidol University:

http://www.sc.mahidol.ac.th/research/vectors.htm PAHO:

http://www.paho.org/english/ad/dpc/cd/dengue.htm

Walter Reed Army Institute of Research:

http://wrair-www.army.mil

WHO DengueNet:

http://www.who.int/csr/disease/dengue/denguenet

Access to this links box is available online. 